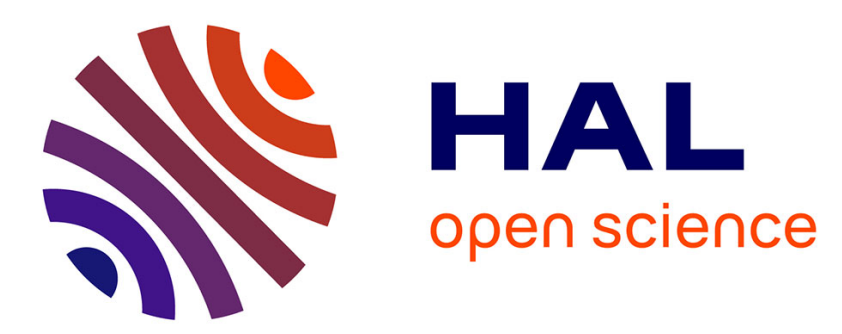

\title{
Modelling and Simulation of a Two wheeled vehicle with suspensions by using Robotic Formalism
}

Salim Maakaroun, Philippe Chevrel, Maxime Gautier, Wisama Khalil

\section{To cite this version:}

Salim Maakaroun, Philippe Chevrel, Maxime Gautier, Wisama Khalil. Modelling and Simulation of a Two wheeled vehicle with suspensions by using Robotic Formalism. IFAC World Congress, Aug 2011, Milano, Italy. hal-00583154

\section{HAL Id: hal-00583154 https://hal.science/hal-00583154}

Submitted on 5 Apr 2011

HAL is a multi-disciplinary open access archive for the deposit and dissemination of scientific research documents, whether they are published or not. The documents may come from teaching and research institutions in France or abroad, or from public or private research centers.
L'archive ouverte pluridisciplinaire HAL, est destinée au dépôt et à la diffusion de documents scientifiques de niveau recherche, publiés ou non, émanant des établissements d'enseignement et de recherche français ou étrangers, des laboratoires publics ou privés. 


\title{
Modeling and Simulation of a Two wheeled vehicle with suspensions by using Robotic Formalism
}

\author{
Salim Maakaroun*+, Philippe Chevrel*+ \\ Maxime Gautier*, Wisama Khalil* \\ *Institut de Recherche en Communications et Cybernétiques de Nantes (IRCCyN) \\ 1,Rue de la Nö̈, B.P. 92101, 44321 Nantes Cedex 3, France \\ (Salim.Maakaroun@irccyn.ec-nantes.fr.Wisama.khalil@irccyn.ec-nantes.fr Maxime.gautier@irccyn.ec-nantes.fr) \\ +Ecole des Mines de Nantes, Département d'Automatique-Productique \\ 4 rue Alfred Kastler - La Chantrerie, B.P. 20722, 44307 Nantes cedex 3, France \\ (Philippe.chevrel@emn.fr)
}

\begin{abstract}
Models, simulators and control strategies are required tools for the conception of secure and comfortable vehicles. The aim of this paper is to present a systematic approach to develop models for dynamic vehicle, focusing on a two wheeled vehicles whose body involves six degrees of freedom. The resulting model is sufficiently generic to perform simulation of realistic cornering and accelerating behaviour in various situations. It may be used in the context of motorcycle modeling, but also in various situations (e.g. for control application) as simplified model for 3 or 4 wheeled (tilting) cars. The approach is based on considering the vehicle as a multi-body poly-articulated system and the modeling is carried out using the robotics formalism based on the modified Denavit-Hartenberg geometric description. In that way, the dynamic model is easy to implement and the system can be used for control applications.
\end{abstract}

Keywords: Modeling, Simulation, Automotive, Robotics, Simulators, Dynamics, Vehicle

\section{INTRODUCTION}

Modeling and simulating vehicle dynamics are fundamental tools for vehicles research and development. They allow understanding the dynamics of vehicles and improving the design in order to ensure the major challenge of having safe, comfortable and economic vehicles. Hence, the goal is to build a mathematical model that illustrates significant aspects of the physical dynamics and then facilitate performance analysis and assess design tradeoffs.

In the literature, most of the models proposed are developed for control applications (Sharp, 1971). They are centred on motorcycle behaviours, and neglect some essential aspects such as gyroscopic effect on the steering handle bar or pitch motion due to the suspension system (Weir 1978), (Katayama et al, 1985).

Lately, some advanced models have been developed using multi body systems. R.S Sharp provided his model by using Autosim software with a description lack on the applied method (Sharp et al, 2001). Later, Cossalter et al developed a model based on Lagrange Formalism that consist on interconnected rigid bodies together with suspensions and other flexible components, supplemented by sophisticated tire and engine models (Cossalter et al, 2002). This formulation uses absolute coordinates that do not depend on the topological structure of the system. However, this technique leads to a complicated model, hard to implement and requires complex numerical algorithm to solve the DAE's (Shabana, 1994).

Therefore to model a complex system (Rajamani, 2006), (Kiencke et al, 2000) in 3D motion, we claim that it is preferable to proceed in a systematic geometrical description, based on the modified Denavit Hartenberg parameterization (Khalil et al 1986). This description allows to automatically calculate the symbolic expression of the geometric, kinematic and dynamic models by using a symbolic software package as SYMORO+ (Symbolic Modeling of Robots) (Khalil et al, 1997). This formulation leads to a minimum set of differential equations from where the constraint equations for the mechanical system are automatically eliminated.

This paper concentrates on developing a dynamical model for a two wheeled vehicle (called bicycle) by applying recursive methods used in robotics. The approach elaborates systematically the symbolic equations of motion and makes the implementation of the dynamic model easier.

This work can be extended for various complex vehicles, such as narrow electric tilting car, specifically Smera Car from Lumeneo (Lumeneo) and (Maakaroun, 2010a, b).

The paper is organized as follows: the global method is described in section 2.1 and applied to the bicycle system in section 2.2. A dynamic model is then elaborated using a recursive Newton-Euler based Algorithm (Khalil et al, 1987) in section 3. Finally, Simulations results are illustrated and commented and conclusions are done. The paper ends with a summarize conclusion.

\section{GEOMETRICAL DESCRIPTION OF THE CAR}

\subsection{Robotic representation of a multi body system}

The bicycle is considered as a mobile robot which is a treestructured multi body system composed of $n$ bodies (links) where the chassis is the mobile base and the wheels are the 
terminal links. The links are numbered consecutively from the base to the terminal links. Each body $C_{j}$ is connected to its antecedent $C_{i}(\mathrm{i}=\mathrm{a}(\mathrm{j}))$ with a joint that represents a translational or rotational degree of freedom and can be elastic or rigid. $a(j)$ denotes the link antecedent to link $\mathrm{j}$, and consequently $\mathrm{a}(\mathrm{j})<\mathrm{j}$.A body can be virtual or real; the virtual bodies are introduced to describe joints with multiple degrees of freedom like ball joint or intermediate fixed frames.

The frame $R_{\mathrm{i}}\left(O_{i}, x i, y_{i}, z_{i}\right)$ which is attached to the body $\mathrm{Ci}$ is defined as following:

The $z_{i}$ axis is along the axis of joint $i$, the $u_{j}$ axis is defined as the common normal between $z_{i}$ and $z_{j}$. The $x_{i}$ axis is along the common normal between $\mathrm{z}_{\mathrm{i}}$ and one of the succeeding $z$ axis, where link $\mathrm{i}$ is the antecedent of link $\mathrm{j}$ and the origin $O_{i}$ is the intersection of $z_{i}$ and $x_{i}$.

The homogeneous transformation matrix ${ }^{i} T_{j}$ between two consecutive frames $R_{i}$ and $R_{j}$ is expressed as a function of the following six parameters (Fig. 1):

- $\gamma_{j}$ : angle between $x_{i}$ and $u_{j}$ about $z_{i}$

- $b_{j}$ : distance between $x_{i}$ and $u_{j}$ along $z_{i}$

- $\alpha_{j}$ : angle between $z_{i}$ and $z_{j}$ about $u_{j}$

- $d_{j}$ : distance between $z_{i}$ and $z_{j}$ along $u_{j}$

- $\theta_{j}$ : angle between $u_{j}$ and $x_{j}$ about $z_{j}$

- $r_{j}$ : distance between $u_{j}$ and $x_{j}$ along $z_{j}$

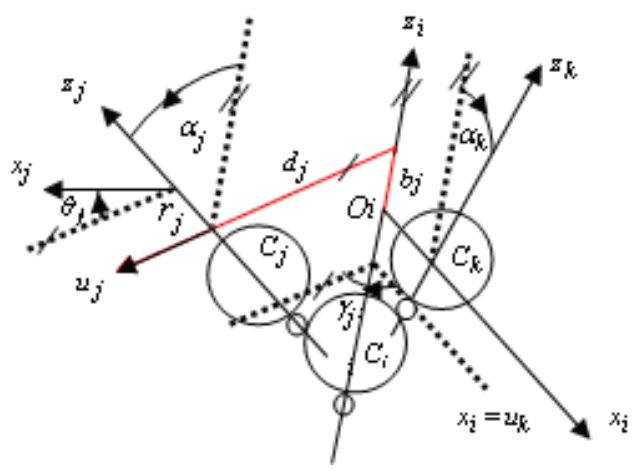

Fig. 1. Geometric parameters

The generalized coordinate of joint $j$ is denoted by $q_{j}$, it is equal to $r_{j}$ if $j$ is translational and $\theta_{j}$ if $j$ is rotational. In (Fig.1), since $x_{\mathrm{i}}$ is taken along $u_{\mathrm{k}}$, the parameters $\gamma_{\mathrm{k}}$ and $b_{\mathrm{k}}$ are equal to zero. We define the parameter $\sigma_{\mathrm{j}}=1$ if joint $j$ is translational and $\sigma_{\mathrm{j}}=0$ if joint $j$ is rotational. If there is no degree of freedom between two frames that are fixed with respect to each other, we take $\sigma_{\mathrm{j}}=2$. In this case, the time derivative of $q_{\mathrm{j}}$ is zero.

\subsection{Application for the model}

Our model is composed of 12 bodies (Fig.2 \& Fig.3)

connected by 11 joints:

- $C_{l}$ is the chassis

- $C_{3}$ and $C_{9}$ are the front and rear suspensions. Their movement is represented by prismatic flexible joints. - $C_{6}$ is the rear driving wheel and $C_{11}$ is the front steering wheel.

- $C_{4}$ is the steering column

- $C_{2}, C_{5}, C_{7}, C_{8}, C_{10}, C_{12}$ are virtual bodies fixed to other links by blocked joints.

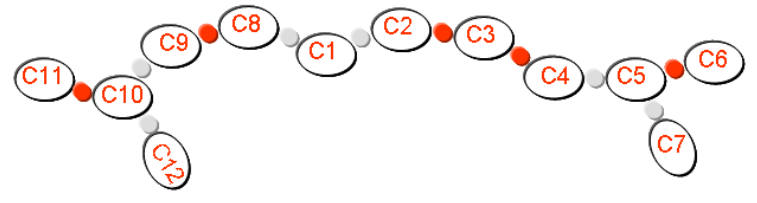

Fig. 2. Multi body description of the bicycle

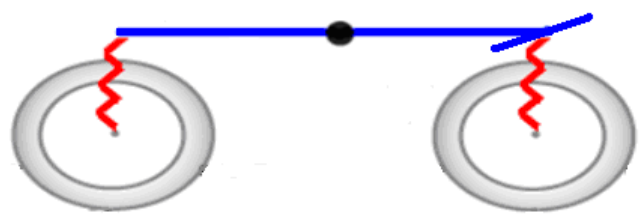

Fig. 3. Shape of the bicycle

The description of the bicycle considered as a multi body poly articulated system uses the Denavit and Hartenberg (MDH) notations that are commonly used in robotics (Fig.4). The chassis motion is described with Euler coordinates while all the other links are described with the generalized Lagrangien coordinates.

According to $\mathrm{MDH}$ description and $\mathrm{SYMORO}+, C_{0}$ is the base attached to the ground. The structure is defined as a robot with a mobile base by considering $C_{1}$ attached to $C_{0}$ via a blocked joint. The inertial parameters of this base are those of $C_{1}$ and the speed and the acceleration are then the ones of the chassis described in his own frame.

Let $R_{\mathrm{f}}$ be a fixed reference frame attached to the ground. The body $C_{1}$ with a location $\zeta$ (i.e. position $\&$ orientation) gives the system posture in the frame $R_{\mathrm{f}}$.

The movement of the chassis in this mixed Euler-Lagrangien model is given by:

$$
{ }^{1} \dot{V}_{1}=\left[\begin{array}{c}
{ }^{1} \dot{V}_{x 1} \\
{ }^{1} \dot{V}_{y 1} \\
{ }^{1} \dot{V}_{z 1}
\end{array}\right],{ }^{1} \omega_{1}=\left[\begin{array}{c}
{ }^{1} \omega_{x 1} \\
{ }^{1} \omega_{y 1} \\
{ }^{1} \omega_{z 1}
\end{array}\right],{ } \dot{\omega}_{1}=\left[\begin{array}{c}
1 \\
\dot{\omega}_{x 1} \\
{ }^{1} \dot{\omega}_{y 1} \\
{ }^{1} \dot{\omega}_{z 1}
\end{array}\right]
$$

Where $\mathrm{V}_{\mathrm{x} 1}, \mathrm{~V}_{\mathrm{y} 1}$ and $\mathrm{V}_{\mathrm{z} 1}$ are respectively the longitudinal, lateral and vertical translational speed of the chassis. Where $\omega_{1}$ and $\dot{\omega}_{1}$ are the angular velocity and accelerations of the chassis.

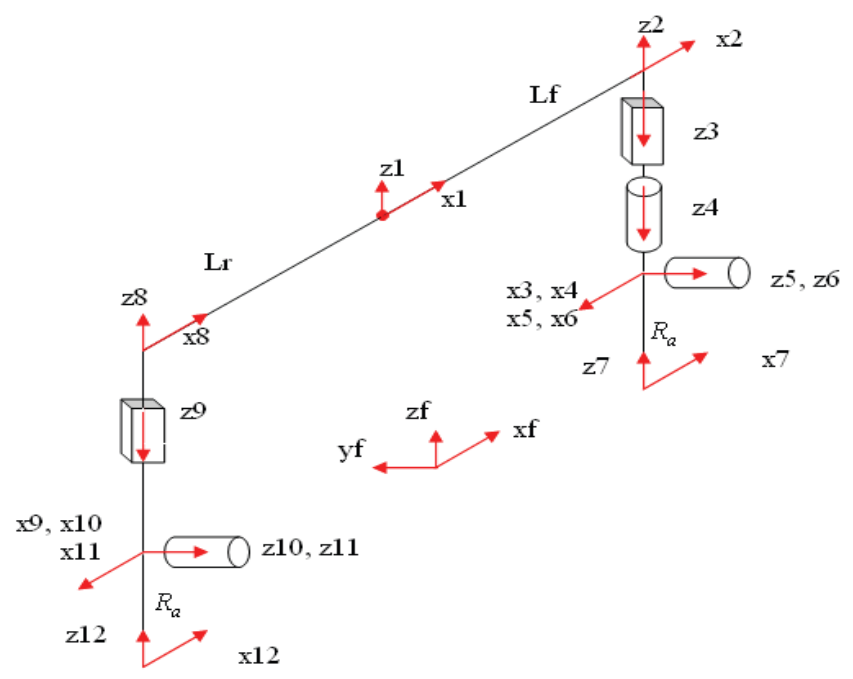

Fig. 4. Geometric description of the bicycle 
According to this description, the geometric parameters of the tree structure are shown in table 1 and the bicycle motion is completely described by the vector $\mathrm{q}$ of the 11 generalized coordinates:

$$
q=\left[\begin{array}{ll}
\xi & \xi_{1}
\end{array}\right]^{T} ; \xi_{1}=\left[\begin{array}{lllll}
r_{3} & r_{9} & q_{6} & q_{11} & q_{4}
\end{array}\right]
$$

- $\xi[1 \times 6]$ is the posture of the chassis (position \& orientation)

$-r_{3}$ and $r_{9}$ are the length of the suspensions,

- $q_{6}$ and $q_{11}$ are the angular positions of the two wheels with respect to their revolute axis,

$-q_{4}$ is the steering angle.

\section{Table 1. Geometric Parameters of the modal}

\begin{tabular}{ccccccccc}
\hline \hline $\boldsymbol{j}$ & $\mathbf{a}(\mathbf{j})$ & $\boldsymbol{\sigma}_{\mathbf{j}}$ & $\boldsymbol{\gamma}_{\mathbf{j}}$ & $\mathbf{b}_{\mathbf{j}}$ & $\boldsymbol{\alpha}_{\mathbf{j}}$ & $\mathbf{d}_{\mathbf{j}}$ & $\boldsymbol{\theta}_{\mathbf{j}}$ & $\mathbf{r}_{\mathbf{j}}$ \\
$\mathbf{1}$ & 0 & 2 & 0 & 0 & 0 & 0 & 0 & 0 \\
$\mathbf{2}$ & 1 & 2 & 0 & 0 & 0 & $\mathrm{~L}_{\mathrm{f}}$ & 0 & 0 \\
$\mathbf{3}$ & 2 & 1 & 0 & 0 & $\pi$ & 0 & $\pi$ & $\mathrm{r}_{3}$ \\
$\mathbf{4}$ & 3 & 0 & 0 & 0 & 0 & 0 & $\mathrm{q}_{4}$ & 0 \\
$\mathbf{5}$ & 4 & 2 & 0 & 0 & $\pi / 2$ & 0 & 0 & 0 \\
$\mathbf{6}$ & 5 & 0 & 0 & 0 & 0 & 0 & $\mathrm{q}_{6}$ & 0 \\
$\mathbf{7}$ & 5 & 2 & 0 & 0 & $\pi / 2$ & 0 & $\pi$ & $-\mathrm{R}_{\mathrm{a}}$ \\
$\mathbf{8}$ & 1 & 2 & 0 & 0 & 0 & $-\mathrm{L}_{\mathrm{r}}$ & 0 & 0 \\
$\mathbf{9}$ & 8 & 1 & 0 & 0 & $\pi$ & 0 & $\pi$ & $\mathrm{R}_{9}$ \\
$\mathbf{1 0}$ & 9 & 2 & 0 & 0 & $\pi / 2$ & 0 & 0 & 0 \\
$\mathbf{1 1}$ & 10 & 0 & 0 & 0 & 0 & 0 & $\mathrm{q}_{11}$ & 0 \\
$\mathbf{1 2}$ & 10 & 2 & 0 & 0 & $\pi / 2$ & 0 & $\pi$ & $-\mathrm{R}_{\mathrm{a}}$
\end{tabular}

\section{DYNAMIC MODEL}

\subsection{Dynamic parameters}

For each link there are 14 standard dynamic parameters (Gautier et al, 1990) composed of 10 standard inertial parameters (Table 2):

- $J_{\mathrm{j}}=\left[X X_{\mathrm{j}} X Y_{\mathrm{j}} X Z_{\mathrm{j}} Y Y_{\mathrm{j}} Y Z_{\mathrm{j}} Z Z_{\mathrm{j}}\right]$ : the six coefficients of the

inertia matrix of link $j$ given in the frame $R_{\mathrm{j}}$,

- $M S_{\mathrm{j}}=\left[M X_{\mathrm{j}} M Y_{\mathrm{j}} M Z_{\mathrm{j}}\right]$ : the three components of first moment

of link $j$ around the origin of the frame $j$,

- $M_{\mathrm{j}}$ : the mass of link $j$

\section{Table 2. Dynamic parameters of the modal}

\begin{tabular}{ccccccccccc}
\hline \hline $\mathbf{j}$ & $\mathbf{X X}$ & $\mathbf{X Y}$ & $\mathbf{X Z}$ & $\mathbf{Y Y}$ & $\mathbf{Y Z}$ & $\mathbf{Z Z}$ & $\mathbf{M X}$ & $\mathbf{M Y}$ & $\mathbf{M Z}$ & $\mathbf{M}$ \\
\hline $\mathbf{1}$ & $\mathrm{XX}_{1}$ & $\mathrm{XY}_{1}$ & $\mathrm{XZ}_{1}$ & $\mathrm{YY}_{1}$ & $\mathrm{YZ}_{1}$ & $\mathrm{ZZ}_{1}$ & $\mathrm{MX}_{1}$ & $\mathrm{MY}_{1}$ & $\mathrm{MZ}_{1}$ & $\mathrm{M}_{1}$ \\
$\mathbf{2}$ & 0 & 0 & 0 & 0 & 0 & 0 & 0 & 0 & 0 & 0 \\
$\mathbf{3}$ & 0 & 0 & 0 & 0 & 0 & 0 & 0 & 0 & 0 & $\mathrm{M}_{3}$ \\
$\mathbf{4}$ & 0 & 0 & 0 & 0 & 0 & 0 & 0 & 0 & 0 & 0 \\
$\mathbf{5}$ & 0 & 0 & 0 & 0 & 0 & 0 & 0 & 0 & 0 & 0 \\
$\mathbf{6}$ & $\mathrm{XX}_{6}$ & 0 & 0 & $\mathrm{YY}_{6}$ & 0 & $\mathrm{ZZ}_{6}$ & 0 & 0 & 0 & $\mathrm{M}_{6}$ \\
$\mathbf{7}$ & 0 & 0 & 0 & 0 & 0 & 0 & 0 & 0 & 0 & 0 \\
$\mathbf{8}$ & 0 & 0 & 0 & 0 & 0 & 0 & 0 & 0 & 0 & 0 \\
$\mathbf{9}$ & 0 & 0 & 0 & 0 & 0 & 0 & 0 & 0 & 0 & $\mathrm{M}_{9}$ \\
$\mathbf{1 0}$ & 0 & 0 & 0 & 0 & 0 & 0 & 0 & 0 & 0 & 0 \\
$\mathbf{1 1}$ & $\mathrm{XX}_{11}$ & 0 & 0 & $\mathrm{YY}_{11}$ & 0 & $\mathrm{ZZ}_{11}$ & 0 & 0 & 0 & $\mathrm{M}_{11}$ \\
$\mathbf{1 2}$ & 0 & 0 & 0 & 0 & 0 & 0 & 0 & 0 & 0 & 0
\end{tabular}

For each actuated joint $j$, we introduce:
- $I_{\mathrm{aj}}$ as the total inertia of the rotor of motor and the drive transmission.

- $F_{\mathrm{vj}}, F_{\mathrm{sj}}$ as the viscous and coulomb friction parameters.

For a flexible joint, we define:

- $K_{\mathrm{j}}$ as the stiffness of the joint $j$

For joint 3 and 9 we add $K_{3}, K_{9}, F_{\mathrm{v} 3}$ and $F_{\mathrm{v} 9}$ to the parameters listed in Table 2 . These parameters represent respectively the stiffness of the springs and the dampers of the suspensions.

\subsection{External Forces}

The external forces applied to the bicycle, which have the most significant impact on vehicle dynamics, are the contact forces between the ground and the tires. These external forces can be modeled (Pacejka, 2002), estimated (Canudas, 2003) or measured at the center of the wheels by using dynamometric wheels.

Aerodynamic forces also have an effect on the vehicle behavior, particularly at high speed $(>90 \mathrm{Km} / \mathrm{h})$.

\subsection{Euler-Lagrange Dynamic model}

The mixed Euler-Lagrange model is obtained from two recurrences of the algorithm of Newton-Euler in the following way (Khalil, 2002):

The forward recursive equations can be summarized as follows: for $\mathrm{j}=1$ to $n$, we calculate the total forces and moments on each link

$$
\begin{aligned}
& { }^{j} \omega_{i}={ }^{j} A_{i}{ }^{i} \omega_{i} \\
& { }^{j} \omega_{j}={ }^{j} \omega_{i}+\bar{\sigma}_{j} \dot{q}_{j}{ }^{j} a_{j} \\
& { }^{j} \dot{\omega}_{i}={ }^{j} A_{i}{ }^{i} \dot{\omega}_{i}+\bar{\sigma}_{j}\left[\ddot{q}_{j}{ }^{j} a_{j}+{ }^{j} \omega_{i} \times \dot{q}_{j}{ }^{j} a_{j}\right] \\
& { }^{j} \dot{V}_{j}={ }^{j} A_{i}\left({ }^{j} \dot{V}_{i}+{ }^{i} \mathrm{Y}_{i}{ }^{i} P_{j}\right)+\sigma_{j}\left(\ddot{q}_{j}{ }^{j} a_{j}+2{ }^{j} \omega_{i} \times \dot{q}_{j}{ }^{j} a_{j}\right) \\
& { }^{j} F_{j}=M_{j}{ }^{j} \dot{V}_{j}+{ }^{j} \mathrm{Y}_{j}{ }^{j} M S_{j} \\
& { }^{j} M_{j}={ }^{j} J_{j}{ }^{j} \dot{\omega}_{j}+{ }^{j} \omega_{j} \times\left({ }^{j} J_{j}{ }^{j} \omega_{j}\right)+{ }^{j} M S_{j} \times{ }^{j} \dot{V}_{j}
\end{aligned}
$$

The backward recursive equations can be summarized as follows: for $\mathrm{j}=\mathrm{n}$ to 1 , we calculate the forces and moments exerted on body $B_{\mathrm{j}}$ by its antecedent $B_{\mathrm{i}}$.

$$
\begin{aligned}
& { }^{j} f_{j}={ }^{j} F_{j}+{ }^{j} f_{e j}+\sum_{s(j)}{ }^{j} f_{s(j)} \\
& { }^{i} f_{j}={ }^{i} A_{j}{ }^{j} f_{j} \\
& { }^{j} m_{j}={ }^{j} M_{j}+{ }^{j} m_{e j}+\sum_{s(j}\left({ }^{i} A_{s(j)}{ }^{s(j)} m_{s(j)}+{ }^{j} \hat{P}_{s(j)}{ }^{j} f_{s(j)}\right)
\end{aligned}
$$

Where:

- $\omega_{j}$ and $\dot{\omega}_{j}$ are respectively the angular velocity and the angular acceleration of body $\mathrm{j}$.

- $\quad F_{\mathrm{j}}$ and $M_{\mathrm{j}}$ are respectively the total forces and moments applied on the body j with respect to $O_{\mathrm{j}}$.

$$
{ }^{j} Y_{j}={ }^{j} \hat{\dot{\omega}}_{j}+{ }^{j} \hat{\omega}_{j}{ }^{j} \hat{\omega}_{j}
$$

- ${ }^{j} A_{i}$ and ${ }^{j} P_{i}$ are respectively the orientation $3 \times 3$ matrix and the position vector of the origin $O_{\mathrm{i}}$ of frame $R_{\mathrm{i}}$ in $R_{\mathrm{j}}$. 


$$
\text { - }{ }^{j} a_{j}=\left[\begin{array}{lll}
0 & 0 & 1
\end{array}\right]^{T} ; \hat{\omega}=\left[\begin{array}{ccc}
0 & -\omega_{z} & \omega_{y} \\
\omega_{z} & 0 & -\omega_{x} \\
-\omega_{y} & \omega_{x} & 0
\end{array}\right]
$$

- $J_{\mathrm{j}}, M S_{\mathrm{j}}$ and $M_{\mathrm{j}}$ are described in section 3.A

- s(j) indicates the bodies whose antecedent is body $R_{\mathrm{j}}$

- $f_{\mathrm{ej}}$ and $m_{\mathrm{ej}}$ are the external forces and moments applied by body $C_{\mathrm{j}}$ on the environment.

When $\sigma_{\mathrm{j}}$ is equal to 2 (a blocked joint), all terms multiplied by $\sigma_{\mathrm{j}}$ or $\bar{\sigma}_{j}$ are eliminated.

The inverse dynamic model gives the joint torques as a function of the joint coordinates, speeds and accelerations. The joint forces or torques are obtained by projecting $f_{\mathrm{j}}$ or $m_{\mathrm{j}}$ on the joint axis $z_{\mathrm{j}}$ and by taking into account the effects of friction and elasticity as follows:

$$
\begin{aligned}
& \Gamma_{j}=\left(\sigma_{j}{ }^{j} f_{j}+\bar{\sigma}_{j}{ }^{j} m_{j}\right)^{T j} a_{j}+\Gamma_{j}^{f}+\Gamma_{j}^{e} \\
& \Gamma_{j}^{f}=F_{s j} \operatorname{sigh}\left(\dot{q}_{j}\right)+F_{v j} \dot{q}_{j} \\
& \Gamma^{e}{ }_{j}=K_{j} q_{j}+o f f_{j}
\end{aligned}
$$

For the body $C_{1}$ there is no projection on the joint axis, so the equations of the chassis will be represented by the total forces $f_{1}$ and moments $m_{1}$ exerted by link 0 on link 1 . Thus the NE equations of the chassis are expressed in terms of Euler variables $\left[{ }^{1} \dot{V}_{1}, \dot{\omega}_{1},{ }^{1} \omega_{1}\right]$.

$$
\left[0_{6 \times 1}\right]=\left[\begin{array}{c}
{ }^{1} f_{1} \\
{ }^{1} m_{1}
\end{array}\right]
$$

$f_{1}$ and $m_{1}$ are equal to zero because there is no body antecedent to the chassis.

The inverse Dynamic model (IDM) of a tree structure with a mobile base can be written as:

$$
\begin{aligned}
\Gamma & =A\left(q_{t}\right) \ddot{q}_{t}+H\left(q_{t}, \dot{q}_{t}\right)+J^{T}\left(q_{t}\right) f_{e} \\
& =f\left(q_{t}, \dot{q}_{t}, \ddot{q}_{t}, f_{e}, g, F_{s}, F_{v}, K\right)
\end{aligned}
$$

- $A_{(11 \times 1)}$ is the inertial matrix of the system

- $H_{(I I x l)}$ is the vector of centrifugal, Coriolis and gravity terms.

- $J_{(I I x p)}$ is the Jacobian matrix and $J f_{e}$ is the vector of generalized efforts representing the projection of the external forces and torques on the joint axes.

- p: number of the components of $f_{e}$

- $q_{t}, \dot{q}_{t}, \ddot{q}_{t}$ are the angular position, velocity and accelerations

of all the joints including the variables of the chassis such as:

$$
q_{t}=\left[\begin{array}{lllllllllll}
x & y & z & \theta & \varphi & \psi & r_{3} & r_{9} & q_{6} & q_{11} & q_{4}
\end{array}\right]^{T}
$$$$
\dot{q}_{t}=\left[\begin{array}{lllllllllll}
{ }^{1} V_{x 1} & { }^{1} V_{y 1} & { }^{1} V_{z 1} & { }^{1} w_{x 1} & { }^{1} w_{y 1} & { }^{1} w_{z 1} & \dot{r}_{3} & \dot{r}_{9} & \dot{q}_{6} & \dot{q}_{11} & \dot{q}_{4}
\end{array}\right]^{T}
$$$$
\ddot{q}_{t}=\left[\begin{array}{lllllllllll}
{ }^{1} \dot{V}_{x 1} & { }^{1} \dot{V}_{y 1} & { }^{1} \dot{V}_{z 1} & { }^{1} \dot{w}_{x 1} & { }^{1} \dot{w}_{y 1} & { }^{1} \dot{w}_{z 1} & \ddot{r}_{3} & \ddot{r}_{9} & \ddot{q}_{6} & \ddot{q}_{11} & \ddot{q}_{4}
\end{array}\right]^{T}
$$

\section{SIMULATOR}

To predict the behaviour of the bicycle, we made a simulator by using the dynamic model obtained from the equation of
Newton-Euler. The simulator architecture is described in figure 5:

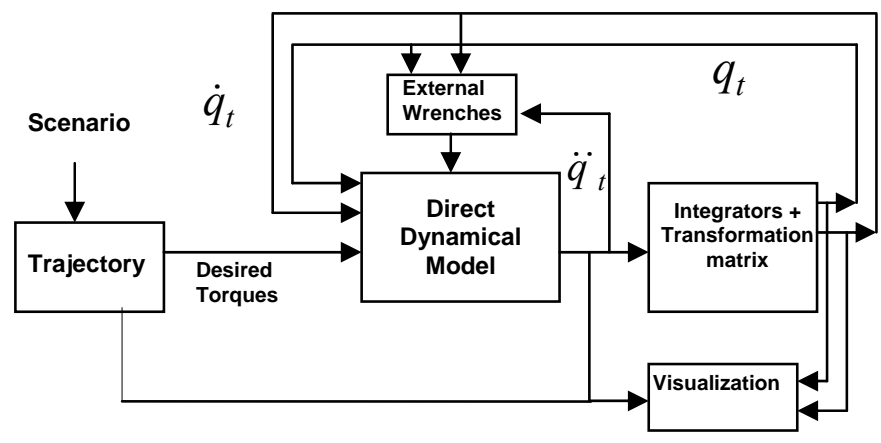

Fig. 5. Simulator architecture

\subsection{Direct Dynamical model}

The direct dynamical model, used in the simulation, gives the joint accelerations as a function of joint positions, velocities torques, and external wrenches. It is represented by the following relation:

$$
\begin{aligned}
\ddot{q}_{t} & =g\left(\Gamma, q_{t}, \dot{q}_{t}, f_{e}, g, F_{s}, F_{v}, K\right) \\
& =\left[A\left(q_{t}\right)\right]^{-1}\left[\Gamma-H\left(q_{t}, \dot{q}_{t}\right)-J^{T}\left(q_{t}\right) f_{e}\right]
\end{aligned}
$$

As we said in Section 3.3, the torques related to the variables of the chassis are zero and the torques of passive joints are zero, we can conclude:

$$
\Gamma(1: 6)=0_{(6 x 1)} \text { and } \Gamma(11)=0
$$

Moreover, by adding the stiffness and the friction coefficient to the suspensions joint as in section 3.1, the torque of joint 3 and 9 will be equal also to zero.

Hence the global vector of torques will be written as follows:

$$
\Gamma=\left[\begin{array}{lllllllllll}
0 & 0 & 0 & 0 & 0 & 0 & 0 & 0 & 0 & \Gamma_{11} & \Gamma_{4}
\end{array}\right]^{T}
$$

The matrix A can be calculated by the algorithm of NewtonEuler, by noting from the relation (8) that the ith column is equal to $\Gamma$ :

$A(:, i)=f\left(q_{t}, 0, u_{i}, 0,0,0,0,0\right)$

$u_{\mathrm{i}}$ is the unit $(11 \times 1)$ vector, whose elements are zero except the ith element which is equal to 1 .

The calculation of the vector $\mathrm{H}$ can be obtained with the Newton-Euler method, by noting that $\mathrm{H}=\Gamma$ if:

$H=f\left(q_{t}, \dot{q}_{t}, 0,0, g, F_{s}, F_{v}, K\right)$

The Matrix $\mathrm{J}^{\mathrm{T}}$ can be calculated as follows:

$J^{T}(:, i)=f\left(q_{t}, 0,0, e_{i}, 0,0,0,0\right)$

$e_{\mathrm{i}}$ is the unit ( $\left.\mathrm{p} \times 1\right)$ vector, whose elements are zero except the ith element which is equal to 1 .

\section{SIMULATION}

In mean to show the behaviour of the bicycle in three dimensions, two test scenarios are considered.

The following assumptions are used:

- The aero dynamical forces are neglected.

-The behaviour of the ground vehicles depends significantly on the nature of the interaction between the tire and the road. As said in section 3.2, this interaction has been modeled and 
the well known and widely used one is Pacejka Magic formula. This model captures in steady-state motion, the tire/road forces and moments, in algebraic equations form with respect to load, longitudinal slip and lateral slip. Among a six components of the tire/road contact wrench, we have considered the principal ones: longitudinal and lateral forces. -To keep the tires in contact with the ground, we must add two constraints to the dynamical model. Therefore, the vertical velocity and acceleration of the contact tire/road with respect to the reference frame must be equal to zero.

$$
\begin{gathered}
{\left[\begin{array}{c}
{ }^{f} V_{7(3)} \\
{ }^{f} V_{12(3)}
\end{array}\right]=\Phi \dot{q}_{t}=0} \\
\frac{d}{d t}\left[\begin{array}{c}
{ }^{f} V_{7(3)} \\
{ }^{f} V_{12(3)}
\end{array}\right]=\frac{d}{d t}\left(\Phi \dot{q}_{t}\right)=\Phi \ddot{q}_{t}+\frac{d}{d t}(\Phi) \dot{q}_{t}
\end{gathered}
$$

Equation (8) becomes:

$$
\Gamma=A\left(q_{t}\right) \ddot{q}_{t}+H\left(q_{t}, \dot{q}_{t}\right)+J^{T}\left(q_{t}\right) f_{e}+\Phi^{T} \lambda
$$

Where:

- $\Phi_{(2 \times 11)}$

- $\quad \lambda_{(2 x I)}$ represents the vector of the efforts transmitted by joints to respect the constraints.

And the direct dynamical model for simulation will be:

$$
\left[\begin{array}{c}
\ddot{q}_{t} \\
\lambda
\end{array}\right]=\left[\begin{array}{cc}
A\left(q_{t}\right) & \Phi^{T} \\
\Phi & 0_{(2 x 2)}
\end{array}\right]^{-1}\left[\Gamma-H\left(q_{t}, \dot{q}_{t}\right)-J^{T}\left(q_{t}\right) f_{e}\right]
$$

\subsection{First Scenario: longitudinal behaviour}

In this scenario, the bicycle is subject to traction torque applied at the rear wheel. The shape of this torque stimulates an accelerated phase then a decelerated one as shown in Fig 6. The trajectory is a straight line with an initial velocity equal to $10 \mathrm{~m} / \mathrm{s}$. When the bicycle accelerates, a transfer load will occur from the front to the rear part of the vehicle. Consequently, the length of the front suspension will increase, and the one of the rear suspension will decrease (Fig 7.). Hence, a negative pitch angle will appear with respect to its revolute axis which is taken to the left as in Fig 8. The velocity and the acceleration of the center of gravity with respect to the reference frame are shown in Fig.9.

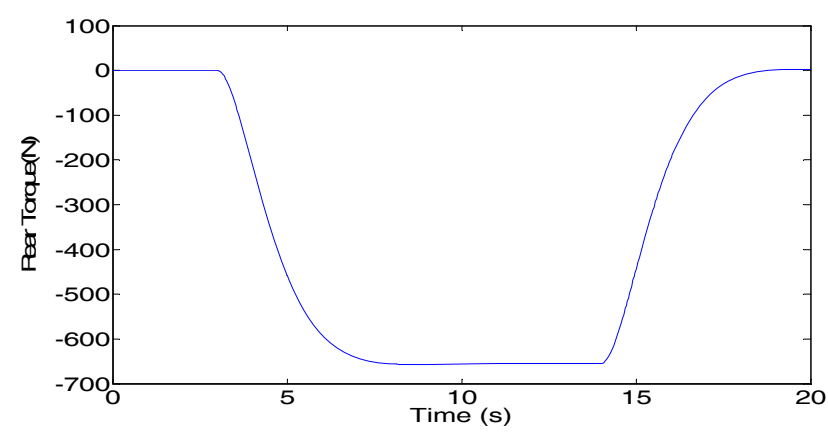

Fig. 6. Rear Torque

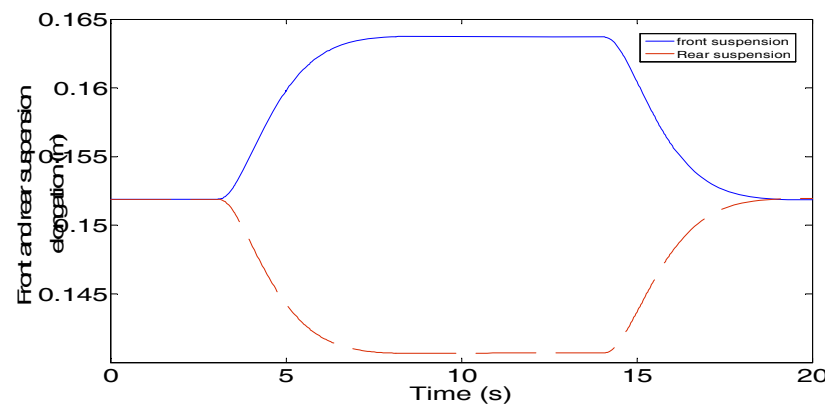

Fig. 7. Front and rear suspension elongation

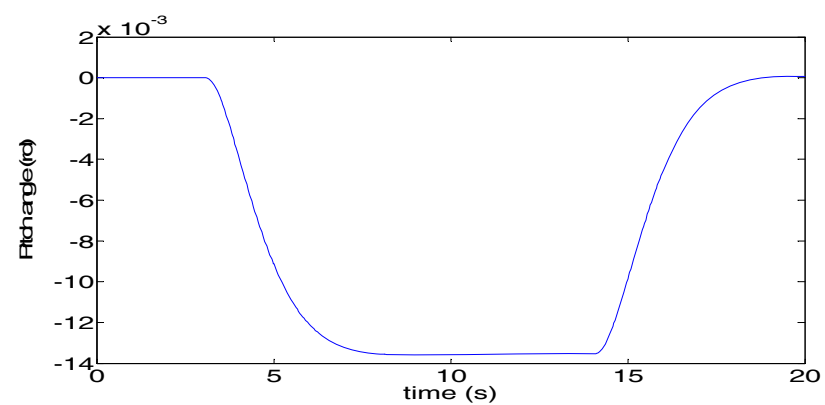

Fig. 8. Pitch angle

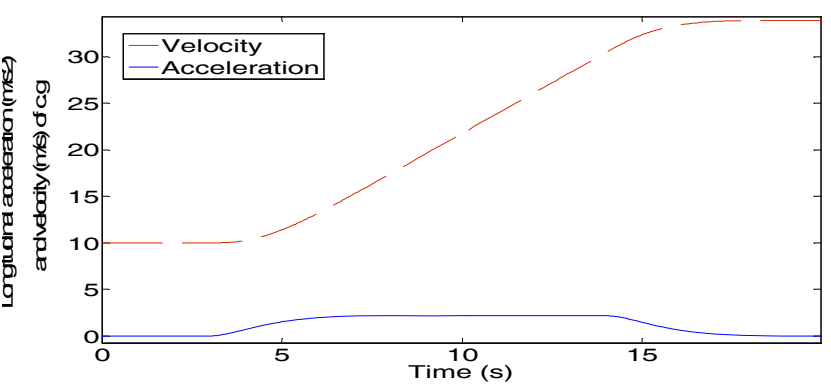

Fig. 9. Longitudinal velocity of c.g

\subsection{Second Scenario: lateral behaviour}

In this scenario, the system is subject to a desired steering torque applied on the steering column to follow the trajectory imposed by a steering desired angle (Fig 10). We consider in this case that the longitudinal velocity is constant and equals to $3 \mathrm{~m} / \mathrm{s}$. However, to maintain the stability, the bicycle must tilt into the corner such that the resultant force of the lateral acceleration and the weight of the vehicle is along the vertical axe of the vehicle. The desired tilt angle will be the roll of the bicycle and it will be equal to:

$$
\theta=a \tan \left({ }^{f} V_{x 1} \dot{\psi} / g\right)
$$

In order to get that, a simple PD controller is used to stabilize the roll dynamics to any desired tilt angle. The controller's output represents the required tilting torque to stabilize the bicycle and it will be applied to the dynamical model by putting it equal to $\Gamma_{5}$.

Fig 10 and Fig 11 illustrate the steering desired angle and the trajectory while the last have the shape of double bend. The roll, yaw and desired tilt angle are shown in Fig. 12 


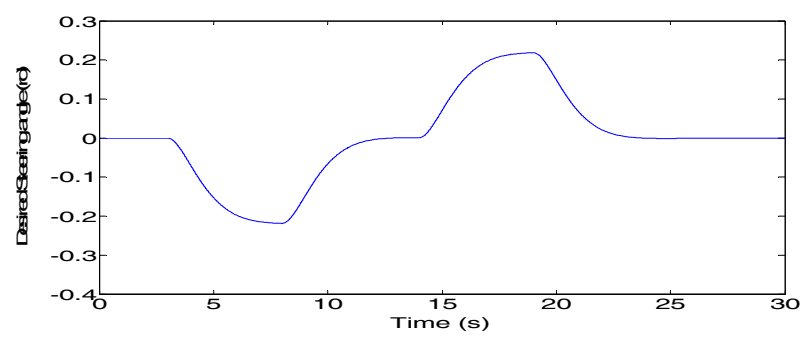

Fig. 10. Desired steering angle

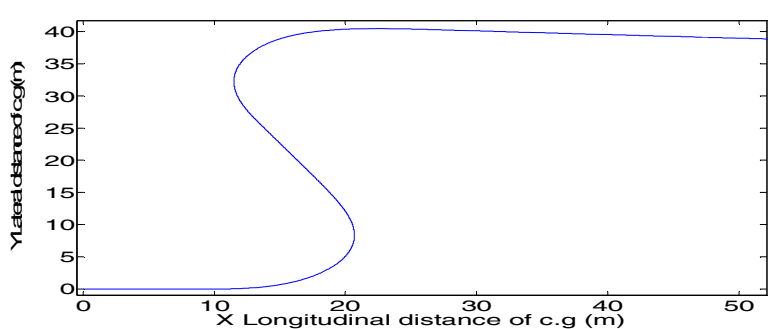

Fig. 11. Trajectory of c.g in the horizontal plan

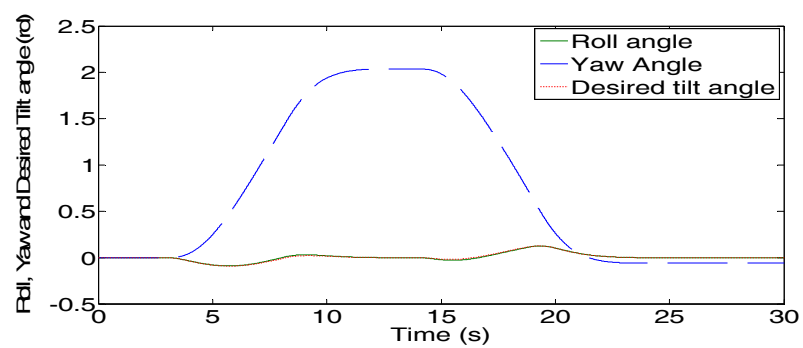

Fig. 12. Roll, Yaw and Desired tilt angle

\section{CONCLUSIONS}

Based on a mixed Euler-Lagrange formulation, a two wheeled vehicle dynamic model was developed for simulation's purpose and some dimensioning and control applications. Future works will consist on applying this technique on more complex and sophisticated vehicles as narrow tilting cars such as the Smera from our collaborator Lumeneo. Two approaches will be compared : i) a bottom up one consisting to analyze the ability of the simplified model proposed in the present paper to give realistic results with ad hoc parameters using identification from experimental data, ii) a top down one, showing how to simplify the complete model for control purpose.

\section{REFERENCES}

Canudas deWit, C., Tsiotras, P. and al, (2003)."Dynamic friction models for road/tire longitudinal interaction," Veh.Syst. Dyn., vol. 39, no. 3, pp. 189-226.

Cossalter, V., Lot, R. (2002). “A motorcycle multi-body model for real time simulations based on the natural coordinates approach," Vehicle System Dynamics, vol. 37, no. 6, pp. 423-447.

Gautier, M. and Khalil, W. (1990), "direct calculations of minimum set of inertial parameters of serial robots," IEEE Trans. On Robotics and Automation, Vol. RA6(3), p. 368-373.

Rajamani R. (2006). vehicle dynamics and control, Springer. Katayama, T., Aoki, A. (1988). "Control Behaviour of
Motorcycle Riders," Vehicle System Dynamics: International Journal of Vehicle Mechanics and Mobility, 17(4), 211-229.

Khalil, W., Kleinfinger, J. (1986). "A new geometric notation for open and closed-loop robots," Robotics and Automation. Proceedings. 1986 IEEE International Confe rence on , vol.3, no., pp. 1174- 1179.

Khalil, W. and Kleinfinger, J.(1987) "Minimum operations and minimum parameters of the dynamic model of tree structure robots,'IEEE J. Robot.Autom., vol. RA-3, no. 6, pp. 517-526.

Khalil, W. and Creusot, D. (1997). "Symoro+: A system for the symbolic modeling of robots," Robotica, vol. 15, no. 2 , pp. $153-161$.

Khalil, W. and Dombre, E. Modeling, Identification and Control of Robots. London : Hermès Penton, 2002.

Kiencke, U. and Nielsen, L. (2000). Automative Control Systems for Engine, Driveline and Vehicle. New York: Springer-Verlag.

Lumeneo, www.lumeneo.fr.

Maakaroun, S, Khalil, W. and al. (2010a). "Geometrical Model of a New Narrow Tilting Car", in Proc. 15th Int Conf. on Methods and Models in Automation and Robotics. Poland

Maakaroun, S, Khalil, W. and al. (2010b). "Dynamic Model of a New Narrow Tilting Car", submitted to. IEEE int Conf. on Robotics and automation, Shanghai

Pacejka. H.B,(2002). Tyre and Vehicle Dynamics. Oxford, U.K.: Butterworth-Heinemann.

Shabana, A. A. (1994). Computational Dynamics. Johan Wiley and Sons.

Sharp, R. S. (1971). "The stability and control of motorcycles," Journal Mechanical Engineering Science, vol. 13 , no. 5 , pp. $316-326$.

Sharp, R. S., (2001). "Stability, control and steering responses of motorcycles," Vehicle System Dynamics, vol. 35(4-5), pp. 291-318.

Weir, D.H. and Zellner, J. W., (1978) "Lateral directional Motorcycle Dynamics and rider control," SAE paper No.780304

\section{Appendix A. SYTEM VARIABLES AND PARAMETERS}

$\mathrm{x}, \mathrm{y}, \mathrm{z}$ : longitudinal, lateral and vertical distance of the centre of gravity with respect to the reference frame.

$\theta, \varphi, \psi$ : roll, pitch and yaw of the bicycle

Ra: Radius of the wheels: $0.275 \mathrm{~m}$

h: $0.625 \mathrm{~m}$ :height of c.g of bicycle from ground

$\mathrm{Lf}=\mathrm{Lr}=0.85 \mathrm{~m}$ : longitudinal distance from c.g to front and rear wheels; $g=9.81 \mathrm{~m} / \mathrm{s}^{2}$ : gravitational constant

$\mathrm{M}_{1}=1000 \mathrm{Kg}$ : mass of the chassis

$\mathrm{M}_{3}=\mathrm{M}_{9}=1.32 \mathrm{Kg}$ : mass of a suspension

$\mathrm{M}_{6}=\mathrm{M}_{11}=10 \mathrm{Kg}$ : mass of a wheel

$\mathrm{XX}_{1}=43.3 \mathrm{Kg} \mathrm{m}^{2}$ : roll moment of inertia of bicycle

$\mathrm{YY}_{1}=254 \mathrm{Kg} \mathrm{m}^{2}:$ pitch moment of inertia of bicycle

$\mathrm{ZZ}_{1}=270 \mathrm{Kg} \mathrm{m}^{2}$ : yaw moment of inertia of bicycle

$\mathrm{XY}_{1}=\mathrm{XZ}_{1}=\mathrm{YZ}_{1}=\mathrm{MX}_{1}=\mathrm{MY}_{1}=\mathrm{MZ}_{1}=0$

$\mathrm{XX}_{6}=\mathrm{YY}_{6}=\mathrm{XX}_{11}=\mathrm{YY}_{11}=0.207 \mathrm{Kg} \mathrm{m}^{2}$

$\mathrm{ZZ}_{6}=\mathrm{ZZ}_{11}=0.378 \mathrm{Kg} \mathrm{m}^{2}$

$\mathrm{K}_{3}=\mathrm{K}_{9}=50000 \mathrm{~N} / \mathrm{m}$ : stiffness of a suspension

$\mathrm{F}_{\mathrm{v} 3}=\mathrm{K}_{\mathrm{v} 9}=5200 \mathrm{Ns} / \mathrm{m}$ : damping coefficient of a suspension 